\title{
Depression in a Real World Population of Hepatitis C Patients
}

\author{
Siddharth Sood ${ }^{1 *}$, Darren Wong ${ }^{1}$, Alex Holmes ${ }^{2}$, Ian Everall ${ }^{2}$, Michael Saling ${ }^{3}$ and Amanda Nicoll ${ }^{1}$ \\ ${ }^{1}$ Department of Gastroenterology and Hepatology, Royal Melbourne Hospital, Parkville, Victoria, Australia \\ ${ }^{2}$ Department of Psychiatry, Royal Melbourne Hospital, Parkville, Victoria, Australia \\ ${ }^{3}$ Melbourne School of Psychological Sciences, University of Melbourne Department of Neuropsychology, Austin Health, Australia
}

Received: June 24, 2014; Accepted: July 20, 2014; Published: July 30, 2014

*Corresponding author: Dr. Siddharth Sood, Gastroenterology Department, Austin Health, Level 8 HSB, Studley Rd, Heidelberg, Victoria, Australia 3084, Tel: +61394965353; Fax: +61394963487;E-mail: Siddharth.sood@austin.org.au

\begin{abstract}
There is evidence as to the neurocognitive effects of the hepatitis $\mathrm{C}$ virus (HCV). In particular, depression is often identified in patients with HCV. Most studies reporting depression rates in HCV populations are in veterans' populations or as part of therapeutic research trials, which represent quite selective populations. In a pilot study, we aimed to determine the frequency of depression in patients with hepatitis $\mathrm{C}$ virus infection attending a liver clinic in Australia. We investigated the records of 100 consecutive HCV patients, documenting an overall rate of depression of $29 \%$, with $19 \%$ on antidepressant medications. A history of intravenous drug use was significantly associated with depression (RR) 2.2 (95\%CI: 1.1-4.4). A history of depression was a significant predictor of depression on treatment with interferon based hepatitis $C$ treatments $(p=0.01)$. Depression is a significant factor in patients attending a liver clinic in Australia. Although newer therapies for hepatitis C may be interferon-free, the high load of mental illness in patients with HCV needs to be considered. The colocation of psychiatric services with hepatology clinics would likely improve patient care.
\end{abstract}

Keywords: Hepatitis C; HCV; Depression; Neurocognition

\section{Introduction}

Hepatitis C virus (HCV) is a common cause of cirrhosis. Without treatment, many patients develop end stage liver disease. Depression and neurocognitive changes have long been associated with patients who have HCV, with recent evidence suggesting the possibility of a direct effect of the virus on the brain and hypothalamic-pituitary axis [1]. Despite the association, few studies have sought to characterise this outside of randomised controlled trials of therapy, which are a much selected subpopulation.

With direct acting antivirals (DAA) offering improved sustained virological response (SVR) rates becoming available, in addition to pegylated interferon and ribavirin, the desire and capacity to treat more patients is of increasing importance. In this pilot study, we aimed to get a real world appreciation for the association between depression and HCV in patients attending liver clinics in Australia. Following on from this pilot study, we aim to undertake future research into depression into patients with HCV and in particular, the presence of genetic markers and the underlying neurocognitive dysfunction that may be present.

\section{Methods}

Investigators performed a file review of 100 patients diagnosed with HCV attending general liver clinics at a tertiary centre in Melbourne, Australia. Demographic details, as well as history of depression, intravenous drug use (IVDU), alcohol use (ETOH) and treatment of HCV were recorded. A random subgroup of patients was referred for neurocognitive testing. Those reporting a current or past history of depression were compared to patients with no history of depression by the chisquared statistics test, with a $\mathrm{p}<0.05$ considered significant. Institutional ethics approval had been granted prior to the study commencing and this research complied with the national statement regarding ethical research in humans.

\section{Results}

Of the 100 patients studied, $76 \%$ were male and the mean age was 54 years (range 22-86). The most common mode of acquisition of $\mathrm{HCV}$ was through intravenous drug use (IVDU) $51 \%$, while $10 \%$ were attributed to blood transfusions and the remainder unknown. A high proportion had a diagnosis of cirrhosis $47 \%$. Year of diagnosis was ascertained in $74 \%$ of patients, with HCV being diagnosed an average 9 years prior to the study.

A prior history of depression was recorded in 29\% of patient histories at baseline, with $19 \%$ on antidepressant medications (ADM) (Figure 1). Depression rates did not vary between men and women. Patients with depression were more likely to have a history of IVDU relative risk (RR) 2.2 (95\%CI: 1.1-4.4), but not alcohol abuse RR 1.6 (95\%CI: 0.8-2.9) or cirrhosis RR 0.8 (95\%CI: 0.4-1.5).

Only 8 patients with a past history of depression had undergone treatment for HCV, compared with 33 patients with no prior history $(28 \% \mathrm{v} 46 \%, \mathrm{p}=0.18$ ). When they did undergo treatment, they were significantly more likely to experience depression during treatment ( $88 \%$ v $27 \%, p=0.01)$. In patients 
with no history of depression, treatment induced depression was noted in $27 \%$ (Table 1), with depression persisting in more than half of patients.

Results on a pilot group of 7 patients with neurocognitive assessment showed reduced processing speed and executive dysfunction, particularly on a measure of rule-based lexical generation.

\section{Discussion}

Depression rates in patients with HCV are increased, but estimates vary similar to other chronic diseases. With much prior research focused on US veteran populations, there is potential for confounding and bias which are not relevant to a real-world population in Australia. We performed a pilot study to evaluate the association between depression and HCV in a real-world setting. On current treatment regimens, a history of significant depression remains a common reason to not treat patients with interferon based therapies [2]. Given the large number of patients with HCV who are not undergoing treatment, and their risk of advancing to cirrhosis and liver-failure, improving treatment rates and outcomes is important. Nearly one third of patients undergoing review in our liver clinics had a past history of depression compared with community prevalence estimated at $10.3 \%$ [3]. Depression appeared to be a deterrent to undergoing treatment for $\mathrm{HCV}$, and with good reason. A pre- existing history of depression remains the most significant risk factor for depression on interferon-based therapies.

Similar to other chronic diseases, neurocognitive symptoms including fatigue and depression appear to be overexpressed in patients with HCV. Neurocognitive dysfunction is present in patients with HCV, even without a diagnosis of cirrhosis or being on treatment. Debate remains whether this is due to HCV being a chronic disease, the presence of associated medical or socio-economic risk factors, or a direct neurocognitive effect of the virus. Early treatment can prevent the development of liver fibrosis and cirrhosis and offers a potential cure, but involves multiple drugs including pegylated interferon which is known to increase depressive symptoms. Recent advancements including newer direct acting antivirals have improved treatment success and offer a greater incentive to treat patients. Physician apprehension regarding a patient's underlying mental illness and its propensity to worsen on HCV treatment is a major factor that limits treatment uptake, especially when limited psychiatric support is available.

In patients with no past history of depression, 1 in 3 patients in our study still experienced depression on interferon based treatment, with half of these experiencing ongoing depressive symptoms despite treatment completion or discontinuation. Again, this may represent an inflated proportion given these patients may have required dose adjustment, drug holidays or

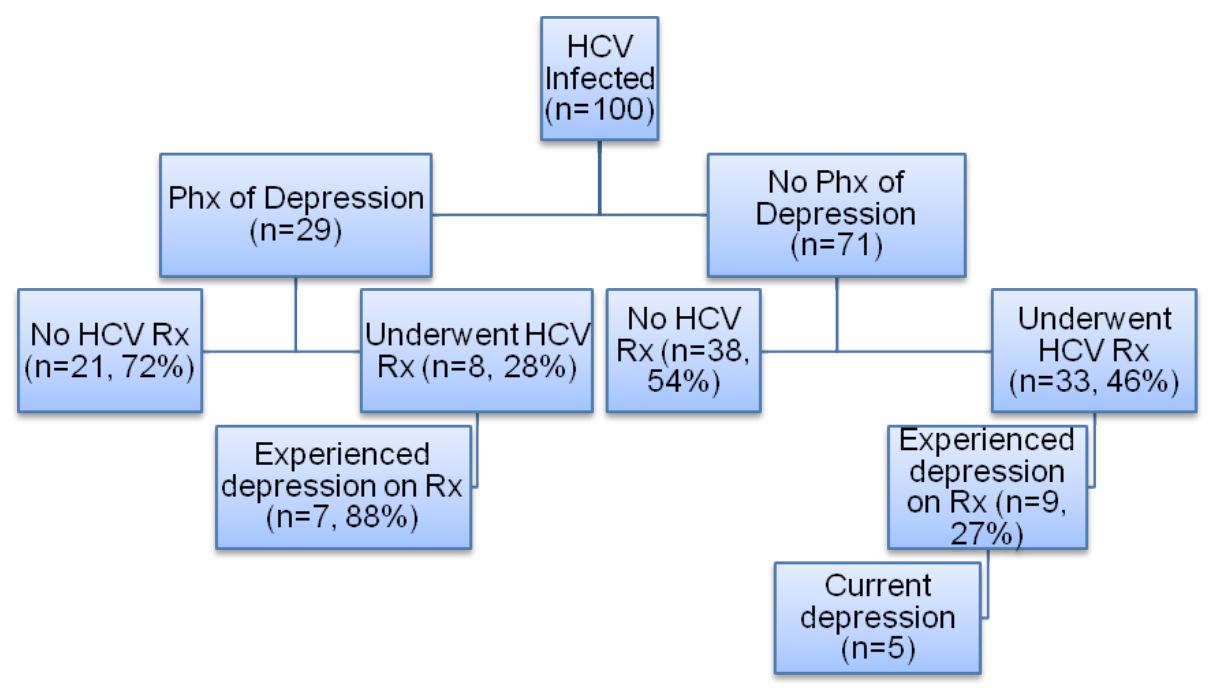

Figure 1: Depression and treatment rates in HCV infected patients.

Table 1: Depression rates during HCV Treatment in non depressed patients $(n=33)$.

\begin{tabular}{|l|c|c|}
\hline & N & \% \\
\hline Depression during treatment & 9 & $27 \%$ \\
\hline ADM ${ }^{*}$ during treatment & 6 & $18 \%$ \\
\hline Current Depression & 5 & $15 \%$ \\
\hline Current ADM* & 3 & $9 \%$ \\
\hline
\end{tabular}

${ }^{*} \mathrm{ADM}=$ Anti depressant medication 
early discontinuation which would increase the risk of treatment failure and need for ongoing clinical review. However, our on treatment depression rate of $27 \%$ is similar to the $26 \%$ noted in a larger study of hepatitis C treatment in nearly 400 patients [4]. However, it reinforces the risks of interferon based therapies on patients experiencing mood disorders and the importance of psychiatric support or co-location with Hepatologists.

Previous estimates of depression in patients with HCV vary significantly from 16-44\% [5-7]. Much variability can be attributed to studies being undertaken in specific subpopulations such as war veterans, IVDU or patients enrolled in drug trials. With this study, we aimed to estimate the frequency of HCV in a local, real-world population. However, this study has obvious limitations. We have undertaken a file review of patients attending a liver clinic, and there is likelihood that patients with depression who were successfully treated have been discharged from ongoing review and not included in analysis. This would indicate the patients who had failed treatment would be increasingly represented in this cohort, as would patients with cirrhosis or other complicated medical problems which may act as a confounder to rates of depression and treatment. Further, a study of 100 patients may be insufficient to determine the true prevalence of depression in HCV patients, but we believe it provides an estimated frequency that highlights the relevance of depression in this patient population with 1 in 3 affected.

Although interferon is associated with worsening of depression on treatment, with good psychiatric care many patients are able to go through standard therapy with minimal symptoms. However, uptake of treatment and clinicians' and patients' willingness to commence treatment remain an issue. The most significant risk for on-treatment depression was a documented past history of depression. This could allow early identification and targeted psychiatric care. However, in hospital mental health services remain under resourced, and in Australia, very few liver clinics have co-location of psychiatric services. Given the high frequency of patients in liver clinics with a history of depression, the co-existence of psychiatrists at liver clinics may be a desirable option to improve both treatment uptake and success.
This study gives a real world appreciation of HCV patients attending an Australian metropolitan liver clinic. Even with the advent of interferon free therapies, depression will remain an ongoing issue in HCV management with a $1 / 3$ of patients having the disease diagnosed at some stage in their past. Future studies will further examine if the HCV virus has a direct effect on neurocognition and depression.

\section{References}

1. Fletcher NF, Wilson GK, Murray J, Hu K, Lewis A, Reynolds GM, et al. Hepatitis $C$ virus infects the endothelial cells of the blood-brain barrier. Gastroenterology. 2012; 142(3): 634-643. DOI: 10.1053/j. gastro.2011.11.028.

2. Guadagnino V, Trotta MP, Carioti J, Caroleo B, Antinori A. Does depression symptomatology affect medication compliance during the first weeks of anti-HCV therapy in intravenous drug users? Digestive and liver disease: official journal of the Italian Society of Gastroenterology and the Italian Association for the Study of the Liver. 2006; 38(2): 119-124. DOI: 10.1016/j.dld.2005.10.008.

3. Goldney RD, Eckert KA, Hawthorne G, Taylor AW. Changes in the prevalence of major depression in an Australian community sample between 1998 and 2008. Aust NZJ Psychiatry. 2010; 44(10): 901-910. DOI: $10.3109 / 00048674.2010 .490520$.

4. Evon DM, Ramcharran D, Belle SH, Terrault NA, Fontana RJ, Fried MW, et al. Prospective analysis of depression during peginterferon and ribavirin therapy of chronic hepatitis C: results of the Virahep-C study. Am J Gastroenterol. 2009; 104(12): 2949-2958. DOI: 10.1038/ ajg.2009.528.

5. Dieperink E, Ho SB, Thuras P, Willenbring ML. A prospective study of neuropsychiatric symptoms associated with interferon-alpha- $2 \mathrm{~b}$ and ribavirin therapy for patients with chronic hepatitis C. Psychosomatics. 2003; 44(2): 104-112.

6. Kraus MR, Schafer A, Faller H, Csef H, Scheurlen M. Psychiatric symptoms in patients with chronic hepatitis $\mathrm{C}$ receiving interferon alfa-2b therapy. J Clin Psychiatry. 2003; 64(6): 708-714.

7. Golub ET, Latka M, Hagan H, Havens JR, Hudson SM, Kapadia F, et al. Screening for depressive symptoms among HCV-infected injection drug users: examination of the utility of the CES-D and the Beck Depression Inventory. J urban Health. 2004; 81(2): 278-290. 